\title{
Re-assessing the ordinary chondrites paradox
}

\author{
T. Mothé-Diniz ${ }^{1}$, F. L. Jasmin ${ }^{2}$, J. M. Carvano ${ }^{2}$, D. Lazzaro ${ }^{2}$, D. Nesvorný ${ }^{3}$, and A. C. Ramirez ${ }^{4}$ \\ 1 Universidade Federal do Rio de Janeiro / Observatório do Valongo Lad.Pedro Antônio, 43 - 20080-090 Rio de Janeiro, Brazil \\ e-mail: thais.mothe@astro.ufrj.br \\ 2 Observatório Nacional, Rua Gal. José Cristino, 77 20921-400 Rio de Janeiro, Brazil \\ e-mail: lazzaro@on.br,flavialuzia@on.br,carvano@on.br \\ 3 Southwest Research Institute, 1050 Walnut St., Suite 300 Boulder, Colorado 80302, USA \\ e-mail: davidn@boulder.swri.edu \\ ${ }^{4}$ Universidad de la Serena Av. Cisternas 1200, La Serena, Chile \\ e-mail: aramirez@dfuls.cl
}

Received 9 December 2009 / Accepted 23 February 2010

\begin{abstract}
Context. The so-called ordinary chondrite paradox has been a recurring topic in planetary science in the past twenty years. This paradox originated from the first comparisons between spectrophotometric measurements of meteorites and asteroids. Basically it is based on the lack of spectral analogs of the ordinay chondrites (OC) among the Main Belt asteroids, although present among the NEAs. Several hypothesis to account for the paradox have been published, all considering the effect of space weathering and different degrees of resurfacing on the asteroids surfaces.

Aims. The aim of the present paper is to further investigate this intriguing problem considering that the small-sized population of Main Belt asteroids has not yet been analyzed.

Methods. Spectroscopic observations of Main Belt asteroids with a size smaller than $5 \mathrm{~km}$ - similar to that of NEAs - were performed with the 8-m Gemini telescope. Furthermore, spectra obtained in large spectroscopic surveys were used to perform a statistical analysis of the fraction of spectral analogous to the OCs in the Main Belt and NEA populations.

Results. The main result of this work is that the vast majority of the Main Belt Sk- and Sq-class asteroids can be matched to OC meteorites in the visible part of the spectrum.

Conclusions. Our results suggest that a considerable fraction of the OC material in the Main Belt is presently unweathered enough to be comparable to meteorite laboratory spectra.
\end{abstract}

Key words. minor planets, asteroids: general - meteorites, meteors, meteoroids

\section{Introduction}

First stated in the 1970, when the first spectroscopic measurements of meteorites were obtained and the first comparisons with spectrophotometric data of asteroids were made (Chapman \& Salisbury 1973), the so-called ordinary chondrite paradox has been a recurring topic in planetary sciences. At that time, spectral analogs for ordinary chondrite meteorites (OC hereafter) were only found among near-earth asteroids (NEAs hereafter). Two taxonomic classes $(\mathrm{O}$ and $\mathrm{Q})$ were then created to represent the asteroids with OC-like visible spectrum. They soon became the synonym for OC-like mineralogies in the literature. Two basic theories were then proposed to explain the apparent absence of OC-mineralogies in the Main Belt: either those mineralogies were in the still unobserved population of the Main Belt, or they look like S-type spectra because of "space weathering" - the change in optical properties of atmosphereless bodies caused by the exposition to the space environment. The main processes that cause space weathering are solar wind implantation and micro-meteorite impacts. Spacecraft observations and laboratory experiments have shown that space weathering can act on asteroid surfaces and change OC-like spectra into S-type spectra (Clark et al. 2002; Chapman 2004; Brunetto \& Strazzulla 2005; Brunetto et al. 2006).

New understandings of the mechanics of the meteorite transport suggested that the meteorite flux could be dominated by a few break-ups near efficient transport routes (Gaffey \& Gilbert 1998; Morbidelli et al. 1994; Migliorini et al. 1997), so that the fall statistics may not be representative of the actual population in the Main Belt. At the same time, mineralogical interpretation of NIR spectra shows that a fraction of the S-type asteroids have mineralogies compatible with OCs (Gaffey et al. 1993), even if their NIR spectra is much redder than the OC laboratory spectra.

These points should have put an end to the paradox. However, the absence of Q-types in the Main Belt, which was interpreted as the absence of unweathered OC-like material, kept this subject alive. At the same time, dedicated surveys of NEAs revealed an increasing number of Q-types in this population (Binzel et al. 2001, 2004). So, the paradox changed from "why don't we have OC-like mineralogies in the Main Belt?" into "why don't we see Q-types (i.e., unweathered OC-surfaces) among Main Belt asteroids?". Two possible explanations rely on the fact that smaller asteroids are observed among NEAs: (1) since they are small, they are less able to retain the regoliths affected by space weathering; or (2) smaller objects have shorter collisional lifetimes, so they would not have time to have their surfaces affected by space weathering.

Further complications to this problem arose from laboratory experiments suggesting that space weathering, in particular what is due to solar wind implantation, could significantly alter the spectra in timescales shorter than the collisional lifetimes of 
the asteroids (Jedicke et al. 2004; Yoshida et al. 2004; Sasaki et al. 2004; Nesvorný et al. 2005; Strazzulla et al. 2005). This prompted an alternative theory proposing that tidal effects of close encounters with the inner planets (Nesvorný et al. 2005; Marchi et al. 2006) could remove the regoliths of small asteroids on shorter timescales than the collisional lifetimes. This theory seemed consistent with a trend of spectral inclinations and perihelion distances seen among NEAs (Marchi et al. 2006). Recently, Binzel et al. (2010) has published a paper that also proposes tidal effects to explain the presence of Q-type asteroids among NEAS.

A central issue here is, however, the assumption that unweathered OC material is seen only among NEAs. This comes from early work that fail to spot asteroids with OC-like spectra in the Main Belt. However, this kind of methodology was not pursued in more recent works. Instead, the association between Q-class spectra and OC-like material seems to have become crystallized in the literature, and the absence of Q-class asteroids in the Main Belt was interpreted as the absence of unweathered OC-like material in this region.

There were several attempts to identify Q-types in the Main Belt, but only recently the first direct OC-matches were found in the Main Belt (Mothé-Diniz \& Nesvorný 2008a), when two authors of the present paper focused the search in small Main Belt asteroid families with formation ages (dated from dynamical methods) shorter than $500 \mathrm{My}$. The authors observed a sample of 6 members of the Datura, Lucascavin, and 1992 YC2 families, obtaining visible spectra in the $0.4-0.92 \mu \mathrm{m}$ range, and used two methodologies to characterize their surface spectra: taxonomy and comparison with meteorite spectra. Out of the six asteroids observed, only one had spectrum compatible with the Q-class, but comparison with meteorites showed that several other asteroids, classified as $\mathrm{Sk}$ or $\mathrm{Sq}$ in the Bus taxonomy, also presented visible spectra that were good matches to several laboratory spectra of OCs.

That result was interpreted as evidence that unweathered OC-like material in the Main Belt could only be found in objects produced by very recent break-ups. However, most of the OC-like spectra found in those families were Sk- and Sq-type, not Q-type, and Sk- and Sq-class asteroids in the Main Belt are not at all rare, even among larger bodies (Bus \& Binzel 2002; Lazzaro et al. 2004).

This understanding led us to the present work. Here we attempt to draw a quantitative picture of the abundance of asteroids with OC-like visible spectra in the Main Belt, and how it compares to what is seen among NEAs. To do so, we resort mostly to literature data, complemented by a spectroscopic campaign made using the Gemini telescope in order to improve the statistics of small asteroids in the Main Belt. These observations, reduction, and classification of the spectra are described in the next section. The selection of the literature sample and the comparison with meteorite spectra are discussed in Sect. 3. From the results drawn there, we proceed to analyze the distribution of asteroids with OC-like spectra in the Main Belt and among NEAs. The results are discussed in Sect. 4.

\section{Observations}

The small Main Belt asteroids selected for observation with the GEMINI telescope had semimajor axes in the range 2.1-2.6 AU, with diameters ranging from 1 to $5 \mathrm{~km}$ (estimated using a mean albedo of 0.15), and visual magnitudes between 17 and 22 . Care was taken in the selection of the objects to (i) avoid sampling objects in the Vesta family, known to have incompatible spectra with OC; and (ii) use color information from the Sloan Digital Sky Survey (Abazajian et al. 2003) to avoid sampling other incompatible spectroscopic types, such as asteroids that could be identified as belonging to the $\mathrm{D}, \mathrm{X}$, and $\mathrm{C}$ taxonomic classes. Observations were performed with the two Gemini Multi-Object Spectrograph (GMOS) (Hook et al. 2004), one at each GEMINI telescope. Observations in the first semester of 2007 (GS-2007A-C-6) were made in classical mode, in three consecutive nights, from 15-17 March, while the programs in the second semester of 2007 (GS-2007B-Q-7,GN-2007B-Q-67) were made in queue mode in the months of August, September, and November, as shown in Table 1 . Both cameras have a pixel size of $0.0727^{\prime \prime} /$ pixel with a field of view of $330 \times 330 \operatorname{arcsec}^{2}$. The spectrographs were used in the long-slit mode with a slit of 1.5". The filter GG455-G0305 was used for the observations, with the grating R150+G5306 for Gemini North and the identical grating R150+G5326 for Gemini South, which yields a useful spectrum ranging from 0.45 to $0.92 \mu \mathrm{m}$. Both GMOS-N and GMOS-S have significant fringing in the red (from $0.7 \mu \mathrm{m}$ on). To minimize this, for every science object we obtained pairs of spectra in two positions of the slit, and performed subtractions B-A. Calibration images of flat fields and arc were obtained via the Gemini Facility Calibration Unit (GCAL), with the same configuration as the science data. After the whole sequence of every object was concluded, three flat-field images and one arc were taken for calibration.

We obtained good $\mathrm{S} / \mathrm{N}$ data for 18 asteroids. A subproduct of this larger project was the analysis of the very young dynamical families (Mothé-Diniz \& Nesvorný 2008b), observed under the GS-2007A-C-6 run. Objects (89309), 2000UV80, and (1226761) were also observed in these programs, but either because of the quality of the spectra, or problems during observing in service mode, they could not be used. All objects were observed with air masses $<1.2$.

\subsection{Reduction and taxonomic classification}

The reduction of the data was made using the standard Gemini IRAF package, with the subpackage GMOS (http: //www .gemini.edu/sciops/data-and-results/ processing-software?q=node/10882). Biases were combined with the task "gbias", flats were combined using the task "gsflat", and science targets were reduced with task "gsreduce", which subtracts the bias, mosaics the three detectors of GMOS, interpolates across the chip gaps for science data, and applies flat-field correction. Then the arc images were also reduced with "gsreduce". For these arcs, the wavelength calibration was established with the task "gswavelength", and the science spectra were further transformed by applying the task "gstransform". Then the sky subtraction was done with "gsskysub", and the pairs of spectra A and B were subtracted, so that we obtained one positive and one negative spectrum. We then extracted all the negative and the positive spectra for every target. The same process was used for the solar analog stars, with the exception of the subtraction A-B.

All spectra of each science target were then summed up, and the same was done for each solar analog. The final spectrum of each asteroid was then extracted and re-binned to match the same points of the summed spectrum of the star. The spectra were then rationed by the solar analog spectrum with air mass closer to the airmass of the target asteroid, and the resulting reflectance spectra were normalized to unity at $0.55 \mu \mathrm{m}$. In Table 1 the details of the observations are presented, such as UT date, 
T. Mothé-Diniz et al.: Re-assessing the ordinary chondrites paradox

Table 1. Observational details for asteroid spectra of the asteroids presented in this paper.

\begin{tabular}{|c|c|c|c|c|c|c|}
\hline Asteroid & UT date & $\begin{array}{c}\text { Exposure } \\
\text { (s) }\end{array}$ & $\begin{array}{l}\text { Mean } \\
\text { air mass }\end{array}$ & $\begin{array}{r}\text { Phase Angle } \\
\text { (deg) }\end{array}$ & Mag. $(V)$ & $\begin{array}{l}\text { Solar } \\
\text { Analog }\end{array}$ \\
\hline$(12210)(1981$ EA42) & 16-Mar.-2007 & $6 \times 250$ & 1.30 & 13.2 & 18.76 & SA104335 \\
\hline$(30173)(2000$ GG72) & 17-Mar.-2007 & $6 \times 250$ & 1.20 & 8.5 & 18.42 & SA104335 \\
\hline (31991)(2000 HK35) & 15-Mar.-2007 & $6 \times 250$ & 1.65 & 10.9 & 18.68 & SA104335 \\
\hline$(36305)(2000 \mathrm{JZ} 56)$ & 17-Mar.-2007 & $8 \times 300$ & 1.43 & 12.4 & 19.25 & SA104556 \\
\hline$(46690)(1997$ AN23) & 16-Mar.-2007 & $6 \times 220$ & 1.63 & 24.9 & 18.85 & SA104335 \\
\hline (48732)(1997 CM4) & 17-Mar.-2007 & $8 \times 260$ & 1.76 & 22.3 & 20.00 & SA104556 \\
\hline$(49985)(2000$ AX1) & 15-Mar.-2007 & $8 \times 240$ & 1.66 & 21.1 & 19.06 & SA104335 \\
\hline$(52349)(1993$ FK15) & 17-Mar.-2007 & $6 \times 280$ & 1.10 & 21.8 & 19.06 & SA104335 \\
\hline$(53769)(2000$ EU85) & 15-Mar.-2007 & $6 \times 250$ & 1.06 & 18.8 & 18.70 & SA104556 \\
\hline (54184)(2000 HJ67) & 15-Mar.-2007 & $6 \times 250$ & 1.27 & 5.3 & 19.03 & SA104556 \\
\hline (55373)(2001 SE240) & 17-Mar.-2007 & $6 \times 290$ & 1.38 & 10.5 & 19.03 & SA104556 \\
\hline$(56447)(2000$ GR76) & 17-Mar.-2007 & $8 \times 250$ & 1.20 & 16.2 & 19.09 & SA104335 \\
\hline (57409)(2001 RT120) & 15-Mar.-2007 & $8 \times 240$ & 1.07 & 14.2 & 19.20 & SA104556 \\
\hline (60645)(2000 FU38) & 15-Mar.-2007 & $8 \times 250$ & 1.60 & 15.3 & 19.24 & SA104335 \\
\hline$(80279)(1999$ XP33) & 17-Mar.-2007 & $8 \times 240$ & 1.80 & 25.4 & 19.03 & SA104456 \\
\hline (85715)(1998 SB54) & 17-Mar.-2007 & $8 \times 250$ & 1.27 & 2.3 & 19.23 & SA104335 \\
\hline (86863)(2000 HY14) & 17-Mar.-2007 & $8 \times 310$ & 1.00 & 20.1 & 19.73 & SA104335 \\
\hline (90127)(2002 XE81) & 16-Mar.-2007 & $10 \times 280$ & 1.90 & 19.1 & 19.40 & SA104335 \\
\hline
\end{tabular}

exposure times, phase angle, apparent magnitude, and the solar analog used for removing the solar flux from the asteroid.

All small objects observed were classified taxonomically according to the algorithm of Bus \& Binzel (2002). In this taxonomic scheme, an asteroid belongs to a given taxonomic class according to the presence or absence of specific features in its visible spectrum, from 0.44 to $0.92 \mu \mathrm{m}$ and to values of particular parameterized spectral characteristics. The taxonomic class attributed to each object is shown in Table 2, where we also list five small main-belt objects observed by Mothé-Diniz \& Nesvorný (2008a), which according to Nesvorný \& Vokrouhlický (2006) belong to very young families (ages $<500 \mathrm{kyr}$ ). Some of the objects had characteristics that were intermediate between two classes of Bus. In this case are the objects (48732), (52349), (54184), and (203370) for which both classes are listed in the table. Table 2 also lists some orbital and physical parameters for each object: semi-major axis $a$, eccentricity $e$, inclination $i$, perihelion distance $q$, and absolute magnitude $H$. Diameters were computed after determining the taxonomic class of the object, using the mean albedo of the corresponding class as listed in Bus (1999). They are 0.15, 0.22, $0.23,0.22,0.29,0.12,0.23$ for the $\mathrm{L}, \mathrm{S}, \mathrm{Sk}, \mathrm{Sl}, \mathrm{Sq}, \mathrm{X}, \mathrm{V}$, respectively. Objects with a double taxonomic class had their diameter estimated using the albedo value for the first class listed. As Bus \& Binzel (2002) does not list albedos for the classes O and Q, we used the value 0.29 to compute diameters of the $\mathrm{O}$ and $\mathrm{Q}$ class objects in our sample. This value corresponds to the mean albedo of the Sq class. The last column of Table 2 lists any dynamical association of each object. We found six objects belonging to the $\mathrm{Q}, \mathrm{Sq}$, or O taxonomic classes.

\section{Literature sample and comparison with meteorites}

To identify the asteroids with unweathered,OC-like spectra, we performed a direct comparison with meteorite spectra from the RELAB database (Pieters \& Hiroi 2004), which presently contains a total of 256 spectra of OC meteorites. We used here the methodology described in (Mothé-Diniz \& Carvano 2005). For consistency, we used a normalization at $0.55 \mu \mathrm{m}$, as is usual in the literature and as the first meteorite-asteroid comparisons were made. The matches with the lower dispersion were subsequently inspected visually. To be deemed a good match, we required that both spectra matched in the region from the maximum that occurs near $0.75-0.8 \mu \mathrm{m}$ to the end of the spectrum at $0.92 \mu \mathrm{m}$. To deal with the inherent subjectiveness of such exercise, the comparison was assessed independently by three different individuals and only the ones that were considered good by at least two were accepted.

At this point we have focused in the classes for which OC spectral analogs have been previously found in the literature: the $\mathrm{Q}$ and $\mathrm{O}$ classes, that have been traditionally associated with OCs, and the Sq and Sk classes, since good OC analogs were found for some asteroids with those classifications by Mothé-Diniz \& Nesvorný (2008b).

Concerning the $\mathrm{Sq}$ class, there were 81 asteroids classified in the S3OS2+SMASSII databases (Bus \& Binzel 2002; Lazzaro et al. 2004), out of which 64 have publicly available spectra in the planetary data system. For the Sk class there are 31 asteroids with this classification, out of which 30 have available spectra. Together with the Gemini spectra of small S-complex asteroids, we have a total of $68 \mathrm{Sq}$ and $33 \mathrm{Sk}$ asteroids that could be compared to the OC meteorite. Out of these, we were able to find good matches to $65 \mathrm{Sq}$ asteroids and $24 \mathrm{Sk}$ asteroids. That is to say, that $95.6 \%$ of the Sq-class and $72.7 \%$ of the Sk-class asteroids in the sample have spectra that is compatible with unweathered OC meteorites. An example of the matches are show in Fig. 1. Table 3 shows the meteorites that are found to be similar to the asteroids, reporting whether it is a "fall" or a "find", the year of recovery, weathering grade, and the number of asteroids found to be similar to each meteorite. The information about the meteorites are from Grady (2000). The information concerning the weathering grade (WG hereafter) is available for only one meteorite: Elenovka, which is a fall, and has WG equal to W0. This means that the meteorite is not altered by terrestrial weathering. Most of the meteorites of Table 3 are also "Fall". According to Wlotzka (1993), it is necessary a minimum of 5000 years of exposure to the terrestrial environments for the meteorite to reach a WG of W2. We therefore assume that terrestrial weathering is not affecting the comparisons. 
Table 2. Physical, orbital, and taxonomic parameters for the objects in the sample.

\begin{tabular}{|c|c|c|c|c|c|c|c|c|}
\hline Asteroid & $a(\mathrm{AU})$ & $e$ & $i$ & $q$ & $H$ & $D$ & Class & Dyn. Family \\
\hline$(12210)(1981$ EA42) & 2.20 & 0.11 & 7.59 & 1.97 & 15.4 & 2.3 & $\mathrm{~V}$ & - \\
\hline$(16598)(1992$ YC2) & 2.62 & 0.22 & 1.63 & 2.05 & 14.7 & 2.8 & $\mathrm{Sq}$ & (16598) \\
\hline$(30173)(2000$ GG72) & 2.34 & 0.01 & 4.72 & 2.31 & 15.2 & 2.6 & $S$ & - \\
\hline (31991)(2000 HK35) & 2.36 & 0.12 & 7.11 & 2.07 & 15.5 & 2.2 & Sk & - \\
\hline$(36305)(2000 \mathrm{JZ} 56)$ & 2.41 & 0.13 & 1.61 & 2.09 & 16.2 & 1.7 & $\mathrm{Sk} / \mathrm{S}$ & - \\
\hline$(46690)(1997$ AN23) & 2.24 & 0.08 & 3.99 & 2.05 & 15.1 & 2.7 & V & - \\
\hline (48732)(1997 CM4) & 2.28 & 0.05 & 6.25 & 2.17 & 14.8 & 2.1 & $\mathrm{Q} / \mathrm{S}$ & - \\
\hline$(49985)(2000$ AX1) & 2.30 & 0.12 & 5.86 & 2.02 & 15.4 & 2.1 & $\mathrm{Sq}$ & - \\
\hline (52349)(1993 FK15) & 2.29 & 0.09 & 4.92 & 2.08 & 15.8 & 2.0 & $\mathrm{~S} / \mathrm{S} 1$ & - \\
\hline (53769)(2000 EU85) & 2.27 & 0.12 & 4.00 & 1.99 & 15.5 & 2.2 & Sl & - \\
\hline$(54184)(2000 \mathrm{HJ} 67)$ & 2.39 & 0.15 & 0.92 & 2.04 & 16.4 & 1.8 & $\mathrm{~L} / \mathrm{S}$ & - \\
\hline$(55373)(2001$ SE240) & 2.41 & 0.15 & 2.72 & 2.05 & 16.4 & 1.5 & $\mathrm{~S}$ & - \\
\hline$(56447)(2000$ GR76) & 2.32 & 0.09 & 5.62 & 2.13 & 15.9 & 1.9 & $\mathrm{~S}$ & - \\
\hline$(57409)(2001$ RT120) & 2.39 & 0.13 & 2.29 & 2.08 & 15.9 & 1.9 & $\mathrm{~S}$ & - \\
\hline (60151)(1999 UZ6) & 2.23 & 0.21 & 5.99 & 1.77 & 16.1 & 1.7 & $\mathrm{Sk}$ & Datura \\
\hline$(60645)(2000$ FU38) & 2.38 & 0.16 & 3.27 & 2.00 & 15.6 & 2.2 & $S$ & - \\
\hline$(80279)(1999$ XP33) & 2.33 & 0.14 & 5.27 & 2.01 & 15.3 & 2.4 & Sl & - \\
\hline (85715)(1998 SB54) & 2.29 & 0.14 & 2.80 & 1.97 & 16.0 & 1.8 & $\mathrm{~S}$ & - \\
\hline$(86863)(2000$ HY14) & 2.29 & 0.18 & 7.11 & 1.88 & 16.3 & 2.1 & $\mathrm{X}$ & - \\
\hline (90127)(2002 XE81) & 2.47 & 0.12 & 6.91 & 2.17 & 15.9 & 1.9 & V & - \\
\hline (90265) (2003 CL5) & 2.24 & 0.21 & 6.00 & 1.77 & 15.4 & 2.1 & $\mathrm{Sq}$ & Datura \\
\hline$(203370)(2001$ WY35) & 2.23 & 0.21 & 5.99 & 1.77 & 17.6 & 0.8 & $\mathrm{O} / \mathrm{Q}$ & Datura \\
\hline$(209570)(2004$ XL40) & 2.28 & 0.11 & 5.98 & 2.02 & 17.1 & 1.0 & $\mathrm{Sq}$ & Lucascavin \\
\hline
\end{tabular}

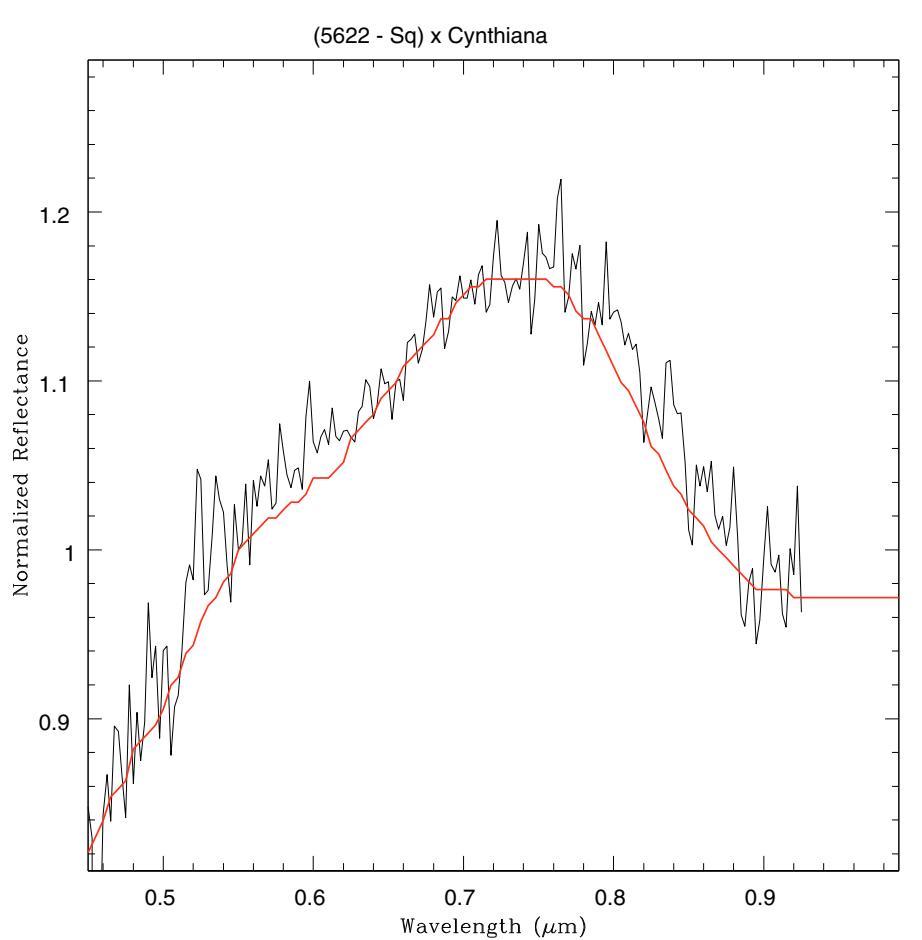

Fig. 1. Spectra of the Sq asteroid (5622) from SMASSII database plotted against the spectrum of the OC meteorite Cynthiana from RELAB, the best match for this asteroid. Both asteroid and meteorite spectra were normalized to unity at $0.55 \mu \mathrm{m}$.

It should be emphasized here that direct comparisons with laboratory meteorite spectra is not suitable to determine the mineralogy of asteroids, since several issues that are not related to mineralogy affect their observed spectra, such as grain size, texture of the surface, shape, solar phase angle. The information that we can extract from such comparisons is just the number of S-complex asteroids that have visible spectra compatible with unweathered ordinary chondrites; that is, the number
Table 3. Meteorites considered the best analogs to S-complex objects analyzed.

\begin{tabular}{llccl}
\hline \hline Meteorite & Fall/Find & Year & WG & Group \\
\hline Bald Mountain & Fall & 1929 & - & L \\
Bison & Find & 1958 & - & LL \\
Bjurböle & Fall & 1899 & - & L/LL \\
Cat Mountain & Find & 1982 & - & L \\
Chainpur & Fall & 1907 & - & LL \\
Cynthiana & Fall & 1877 & - & L/LL \\
Dhajala & Fall & 1973 & - & H \\
Elenovka & Fall & 1951 & Wo & L \\
Forest City & Fall & 1890 & - & H \\
Goodland & Find & 1923 & - & L \\
Hamlet & Fall & 1959 & - & LL \\
Homestead & Fall & 1875 & - & L \\
Knyahinya & Fall & 1866 & - & L/LL \\
Mezö-Madaras & Fall & 1852 & - & L \\
Nuevo Mercurio & Fall & 1978 & - & H \\
Paragould & Fall & 1930 & - & LL \\
Queen's Mercy & Fall & 1925 & - & H \\
Rio Negro & Fall & 1934 & - & L \\
Saratov & Fall & 1918 & - & L \\
Sete Lagoas & Fall & 1908 & - & H \\
Soko-Banja & Fall & 1877 & - & LL \\
Weston & Fall & 1807 & - & H \\
\hline
\end{tabular}

of asteroids whose surfaces do not show any noticeable effect of space weathering in the visible, assuming that all S-complex asteroids have OC-like mineralogies. The reality of this assumption will be discussed in Sect. 4. For now we consider this a working hypothesis and will proceed to analyze the distribution of unweathered OC-like asteroids in the Main Belt and to compare to what is seem among NEAs.

A first issue is to derive the abundance of unweathered material in the Main Belt. We will consider as base of comparison the total number of asteroids with the taxonomic classification that may be compatible with OC mineralogies: the classes Q 
and $\mathrm{O}$ and the $\mathrm{S}$-complex, comprising a total of 793 asteroids (including the Gemini sample). Assuming here that all $\mathrm{O}, \mathrm{Q}$, and, respectively, $95.6 \%$ and $72.7 \%$ of all classified Sq and Sk asteroids are composed of unweathered OC material, we conclude that $15.2 \%$ of the Main Belt asteroids that can have OC-like mineralogies in the SMASS+S3OS2 sample do not show evidence of space weathering. Applying the same reasoning to a sample of NEAs from Binzel et al. (2004), we find that $41 \%$ of the possibly OC-like asteroids in the NEA population are unweathered. While this agrees with the current view that unweathered asteroids are more common among NEAs, the amount of unweathered asteroids in the Main Belt revealed here is considerable.

Next we analyze the distribution of unweathered OC-like asteroids with orbital elements and absolute magnitude and compare it to the reference sample. One problem here is that we do not have spectra for some of the Main Belt asteroids, or for the NEA sample. For the sake of comparison we will assume in the remaining of this section that all $\mathrm{Sq}$ and Sk asteroids have unweathered OC-like composition. The distributions of unweathered OC-like asteroids with semi-major axis, eccentricity, inclinatio, and absolute magnitude were compared to the distributions of the all S-complex asteroids using the Kolmogorov-Smirnov test. The distributions with semi-major axis and inclination for the unweathered OC-like material were not statistically different from the ones of all S-complex, with probabilities of $35.0 \%$ and $87.7 \%$, respectively, that the samples have the same distribution. The distributions of eccentricity and absolute magnitude, however, were considerably different, with probabilities of having the same distribution as the reference sample of $0.2 \%$ and $0.01 \%$, respectively. The distributions for eccentricity is shown in Fig. 2, and the ones for absolute magnitude in Fig. 3. From these histograms it is evident that the Sq-, Sk-, O-, and Q-class asteroids are more frequent at higher eccentricities and absolute magnitudes than the bulk of the S-complex. The same comparison for the NEA sample yields similar results: the distribution for semi-major axis and absolute magnitude for the Sq, Sk, O, and Q asteroids cannot be distinguished from the whole $\mathrm{S}$-complex (probabilities of the distributions being equal of $85.4 \%$ and $56.5 \%$, respectively, but again the distributions for eccentricity and absolute magnitude are clearly different, (probabilities of $0.8 \%$ and $2.6 \%$, respectively), with the Sk, Sq, O, and Q asteroids being more likely to be found at higher eccentricities and less frequently at lower absolute magnitudes than the whole sample.

\section{Discussion and conclusions}

The main result of this work is that the vast majority of the Sk-class and Sq-class Main Belt asteroids can be matched to OC meteorites in the visible part of the spectrum. While this does not guarantee that the similarity would extend into the NIR, such associations are actually not new. Burbine (2000) had already established the similarities of the spectra of some $\mathrm{Sq}$ and Sk asteroids with OC meteorites up to $1.6 \mu \mathrm{m}$.

Even so, the interpretation of the findings in this article depends crucially on what fraction of S-complex asteroids actually have OC mineralogies. If most of S-complex are composed by OC material, as assumed in the previous section, then our results will set new constraints on the interplay between space weathering and the processes that act to refresh the surfaces of asteroids. If on the other hand the fraction of S-complex asteroids with OC mineralogies turn out to be small, then the interpretation of our results is less clear, but the same applies to previous works

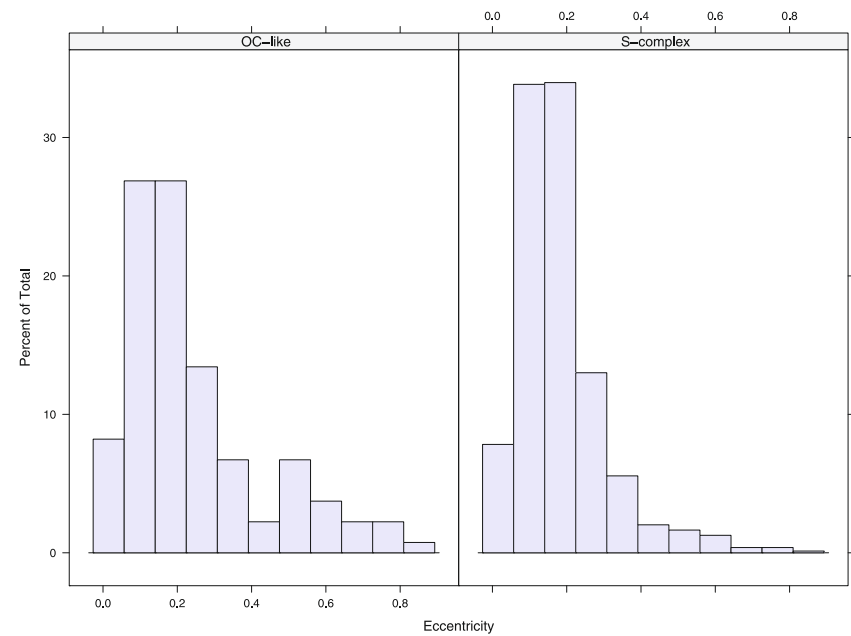

Fig. 2. Histogram comparing the eccentricity distribution of objects in the Main Belt with spectra similar to OC meteorites (left panel) with the eccentricity distribution of all objects belonging to the S-complex.

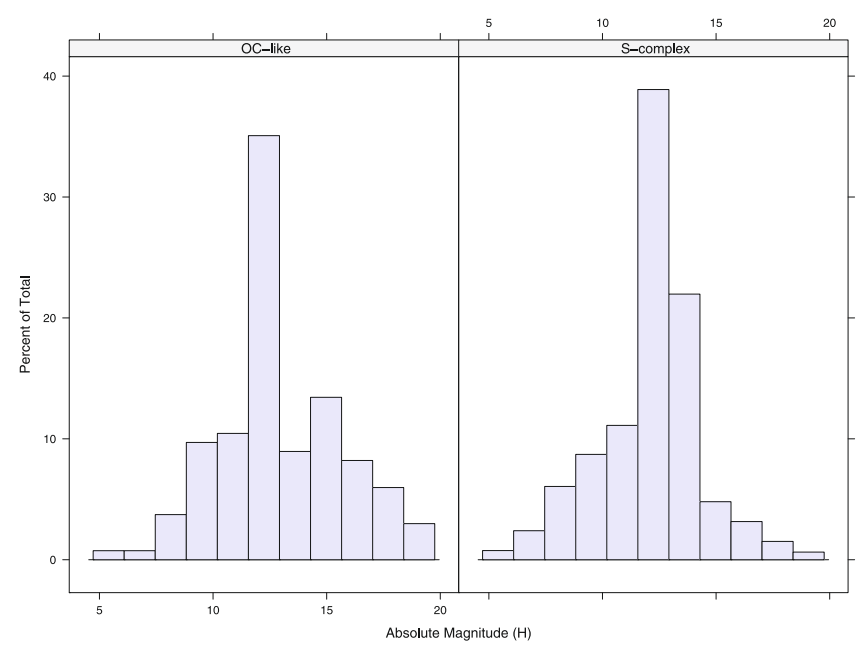

Fig. 3. Same as in Fig. 2, but for the absolute magnitude H.

that deal with the abundance of unweathered OC material among NEAS (Binzel et al. 2004; Marchi et al. 2006).

This issue can only be addressed by the analyzing a large sample of NIR spectra of S-complex asteroids. Gaffey et al. (1993) analyzed a sample of 39 spectra of S-complex asteroids between 0.8 and $2.5 \mu \mathrm{m}$, from the survey conducted by Bell et al. (1988). Based on the position of the 1 and $2 a \mu \mathrm{m}$ bands and on the band area ratio, and using calibrations derived from laboratory measurements of meteorites, the authors conclude that $25 \%$ of their sample were compatible with the OC mineralogy. Vernazza et al. (2007) use a sample of 150 spectra of S-complex asteroids from 0.4 to $2.5 \mu \mathrm{m}$ (but with the visible and NIR portions obtained at different occasions) to investigate this issue. The authors use Shkuratov et al. (1999) radiative transfer model, the Brunetto et al. (2006) space weathering model, and optical constants derived from the work of Lucey (1998) in their analysis. Based mainly on the pyroxene to olivine ratios derived with their methodology, they conclude that more than $70 \%$ of their sample were compatible with OC meteorites. The reasons for this huge discrepancy between the works of Gaffey et al. and Vernazza et al. are difficult to assess, mainly because of the difference in methodologies. One possible explanation is the low emphasis on pyroxene composition given in Vernazza et al., which is constrained by the band positions and laboratory 
calibrations in Gaffey et al. (1993). Recently, however, Gietzen et al. (2008) have suggested that the displacement in the positions of the bands that was attributed by Gaffey et al. as evidence of calcic pyroxene (and interpreted as a product of differentiation) could be produced by non-calcic clinorhombic pyroxenes, which the authors claim to have identified in the spectra of ordinary chondrites using the modified Gaussian model. Clearly more work is needed to clarify this important issue. At this point all that can be said is that the hypothesis that most of the S-class asteroids have OC-mineralogy is admissible, given the difficulty of extracting a clear mineralogical interpretation from the available data, and clearly compatible in terms of pyroxene to olivine ratios alone. It is also interesting to note that 3 out of the four S-type asteroids that were visted by spacecrafts so far had mineralogy compatible with OC meteorites (Clark et al. 2002).

Admitting that this is the case, our results suggest that a considerable fraction of the OC material in the Main Belt is presently sufficiently unweathered to be comparable to meteorite laboratory spectra. This is at odds with the prevailing view that the spectral modifications induced by space weathering occur on much shorter timescales than the collisional refreshment of the asteroid surfaces. Also, the alternative theory of Nesvorný et al. (2005) cannot be invoked here, since Main Belt asteroids do not get close enough to the inner planets for tidal effects to be an issue.

On the other hand, that the unweathered asteroids are more frequently found at higher eccentricities and higher absolute magnitudes is consistent with surface refreshment induced by collisions. Since space weathering tends to lower the albedo, the higher magnitudes suggest smaller asteroids, which are less capable of retaining regoliths. Also, the collision probability is enhanced at higher eccentricities (Farinella \& Davis 1992). Given that a similar behavior is seen among NEAs, it is reasonable to assume that the same mechanism is responsible for the surface renewal on both populations, and it is interesting to note that the higher number of unweathered asteroids at high eccentricities among NEAs might be the culprit for the correlation between spectral inclination and perihelion distance reported by (Marchi et al. 2006) and interpreted as evidence of tidal effects. This may also explain the results of Binzel et al. (2010).

The mismatch between the timescales for spectral modification by space weathering and collisional refreshment remains to be explained. We see two possible explanations here. One is that, for some unidentified reason the laboratory experiments are not reproducing the actual processes that take place on asteroid surfaces, so that the timescale for spectral modification is in fact longer than what is presently accepted. Another is that the relevant timescale for collisional refreshment is not, as widely suggested, the collisional lifetime of the asteroids. Indeed, all that is needed to produce an apparently unweathered asteroid is to remove the thin superficial layer affected by space weathering of a sufficiently large portion of its surface, and that could be accomplished by smaller, non-catastrophic collisions that should occur on shorter timescales. That would be particularly true for smaller asteroids.

Acknowledgements. T.M.-D., J.M.C., D.L. and F.L.J. were supported by various fellowships and grants by CNPq, FAPERJ, and CAPES. D.N. was supported by the NASA's Planetary Geology and Geophysics program, and CNPq.
Based on observations obtained at the Gemini Observatory, which is operated by the Association of Universities for Research in Astronomy, Inc., under a cooperative agreement with the NSF on behalf of the Gemini partnership: the National Science Foundation (United States), the Science and Technology Facilities Council (United Kingdom), the National Research Council (Canada), CONICYT (Chile), the Australian Research Council (Australia), Ministério da Ciência e Tecnologia (Brazil), and Ministerio de Ciencia, Tecnologia e Innovacion Productiva (Argentina).

Data used in this paper were obtained under Program ID: GS-2007A-C-6, GS2007B-Q-7, and GN-2007B-Q-67.

We are grateful to all the staff of GEMINI telescopes who assisted us in the observational programs and especially to Rodrigo Carrasco and Susan Ridgway, who were in direct contact with us during the whole preparation of Phase II and the observations in the classical mode turn, for their patience, and professionalism. We are also greateful to Dr. Andrew Rivkin for the helpful comments and suggestions.

\section{References}

Abazajian, K., Adelman-McCarthy, J. K., Agüeros, M. A., et al. 2003, AJ, 126, 2081

Bell, J. F., Owensby, P. D., Hawke, B. R., \& Gaffey, M. J. 1988, in Lunar and Planetary Institute Conference Abstracts, 57

Binzel, R. P., Harris, A. W., Bus, S. J., \& Burbine, T. H. 2001, Icarus, 151, 139

Binzel, R. P., Rivkin, A. S., Stuart, J. S., et al. 2004, Icarus, 170, 259

Binzel, R. P., Morbidelli, A., Merouane, S., et al. 2010, Nature, 463, 331

Brunetto, R., Romano, F., Blanco, A., et al. 2006, Icarus, 180, 546

Brunetto, R., \& Strazzulla, G. 2005, Icarus, 179, 265

Burbine, T. H. 2000, PhD Thesis, MIT

Bus, S. J. 1999, PhD Thesis, AA(Massachusetts Institute of Technology)

Bus, S. J., \& Binzel, R. P. 2002, Icarus, 158, 106

Chapman, C. R. 2004, Ann. Rev. Earth Plan. Sci., 32, 539

Chapman, C. R., \& Salisbury, J. W. 1973, Icarus, 19, 507

Clark, B. E., Hapke, B., Pieters, C., \& Britt, D. 2002, Asteroids III, 585

Farinella, P., \& Davis, D. R. 1992, Icarus, 97, 111

Gaffey, M. J., Burbine, T. H., Piatek, J. L., et al. 1993, Icarus, 106, 573

Gaffey, M. J., \& Gilbert, S. L. 1998, Meteoritics Planet. Sci., 33, 1281

Gietzen, K. M., Lacy, C. H. S., Ostrowski, D. R., \& Sears, D. W. G. 2008,

in Lunar and Planetary Inst. Technical Report, Lunar Planet. Inst. Sci. Conference Abstracts, 39, 1125

Grady, M. M. 2000, Catalogue of Meteorites (Grady, M. M.)

Hook, I. M., Jørgensen, I., Allington-Smith, J. R., et al. 2004, PASP, 116, 425

Jedicke, R., Nesvorný, D., Whiteley, R., Ivezić, Ž., \& Jurić, M. 2004, Nature, 429,275

Lazzaro, D., Angeli, C. A., Carvano, J. M., et al. 2004, Icarus, 172, 179

Lucey, P. G. 1998, J. Geophys. Res., 103, 1703

Marchi, S., Magrin, S., Nesvorný, D., Paolicchi, P., \& Lazzarin, M. 2006, MNRAS, 368, L39

Migliorini, F., Morbidelli, A., Zappala, V., et al. 1997, Meteoritics Planet. Sci., 32, 903

Morbidelli, A., Gonczi, R., Froeschle, C., \& Farinella, P. 1994, A\&A, 282, 955

Mothé-Diniz, T., \& Carvano, J. M. 2005, A\&A, 174, 54

Mothé-Diniz, T., \& Nesvorný, D. 2008a, A\&A, 492, 593

Mothé-Diniz, T., \& Nesvorný, D. 2008b, A\&A, 486, L9

Nesvorný, D., \& Vokrouhlický, D. 2006, AJ, 132, 1950

Nesvorný, D., Jedicke, R., Whiteley, R. J., \& Ivezić, Ž. 2005, Icarus, 173, 132

Pieters, C. M., \& Hiroi, T. 2004, in Lunar and Planetary Institute Conference Abstracts, 1720

Sasaki, S., Ueda, Y., Loeffler, M., \& Hiroi, T. 2004, in COSPAR, Plenary Meeting, 35th COSPAR Scientific Assembly, 35, 3732

Shkuratov, Y., Starukhina, L., Hoffmann, H., \& Arnold, G. 1999, Icarus, 137, 235

Strazzulla, G., Dotto, E., Binzel, R., et al. 2005, Icarus, 174, 31

Vernazza, P., Binzel, R. P., DeMeo, F. E., \& Thomas, C. A. 2007, BAAS, 38, 476

Wlotzka, F. 1993, Meteoritics, 28, 460

Yoshida, F., Dermawan, B., Ito, T., et al. 2004, PASJ, 56, 1105 